\title{
Femoral nerve palsy as a complication of anterior iliac crest bone harvest: Report of two cases and review of the literature
}

\author{
Jennifer Kargel BS, Vanessa Dimas BS, Wayne Tanaka DDS, O Bailey Robertson DDS, J Michael M Coy DDS, \\ Jack Gotcher DMD PhD, Peter Chang MD DMD
}

\begin{abstract}
J Kargel, V Dimas, W Tanaka, et al. Femoral nerve palsy as a complication of anterior iliac crest bone harvest: Report of two cases and review of the literature. Can J Plast Surg 2006;14(4):239-241.
\end{abstract}

There are many documented neurological complications of anterior iliac crest bone harvest. Until now, these have included injuries to the iliohypogastric, subcostal and lateral femoral cutaneous nerves. Femoral nerve palsy as a direct surgical complication of anterior iliac crest bone harvest has never been cited in any surgical literature, although it has been reported in deep pelvic and abdominal surgeries in which improper retraction and/or prolonged hyperextension of the hip may have caused a nerve compression syndrome. In addition, surgical patients on antithrombolytic therapy have experienced hemorrhage within the iliacus and iliopsoas muscles, resulting in hematoma and secondary femoral nerve compression. The classic motor and sensory deficits reported in femoral nerve palsies are reduced or absent patellar reflex, weak hip flexion, quadriceps muscle weakness, and anesthesia of the anterior thigh and medial aspect of the leg. Two cases of femoral nerve palsy with different etiologies are presented.

Key Words: Bone graft; Complications; Femoral nerve palsy; Iliac crest

Tliac crest bone graft harvesting is a widely used and successful technique for obtaining autologous bone used in the reconstruction of bony defects. Other available sources of bone graft harvesting include the tibia, rib and calvarium. Use of the iliac crest to obtain cortical and cancellous tissue is preferable to these methods for a variety of reasons. The ilium contains abundant amounts of cortical and cancellous bone, which is ideal for bone grafting in most situations. The resulting scar is easily concealed and therefore more aesthetically pleasing to the patient. Because the donor site is located away from the recipient site, this form of bone graft harvesting may be performed simultaneously with the reconstructive operation, which reduces operative time and the risks associated with multiple operations. Postoperatively, patients who undergo this procedure often rapidly return to their routine level of physical activity without significant limitations in the early postoperative period.

With this procedure, as with all invasive operations, there are inherent risks. A review of the literature has shown that the most commonly reported complications are postoperative pain (1-3), seroma or hematoma formation $(2,4)$, wound infection $(1,2,4)$, wound drainage and dehiscence $(5)$, alteration of

\section{La paralysie du nerf fémoral comme complication d'un prélèvement osseux sur la crête iliaque : Rapport de deux cas et analyse bibliographique}

Il existe de nombreuses complications neurologiques documentées de prélèvements osseux sur la crête iliaque. Jusqu'à présent, elles incluaient des lésions aux nerfs iliohypogastrique, sous-costal et cutané fémoral latéral. La paralysie du nerf fémoral comme complication chirurgicale directe d'un prélèvement osseux sur la crête iliaque n'a jamais été citée dans les publications chirurgicales, mais elle l'a été dans le cas d'opérations pelviennes profondes et abdominales au cours desquelles une mauvaise rétractation ou une hyperextension prolongée de la hanche pouvait avoir causé un syndrome de compression nerveuse. De plus des patients opérés prenant des antithrombolytiques ont subi des hémorragies dans les muscles iliaque et psoas-iliaque, qui ont provoqué un hématome et une compression secondaire du nerf fémoral. Les déficits moteurs et sensoriels classiques déclarés en cas de paralysie du nerf fémoral sont une diminution ou une absence du réflexe rotulien, une flexion limitée de la hanche, une faiblesse du muscle quadriceps et une anesthésie de la cuisse antérieure et de l'aspect médial de la jambe. Deux cas de paralysie du muscle fémoral aux étiologies différentes sont présentés.

the superior contour of the iliac crest $(1,3)$, decreased mobility of the thigh, injury of the superior gluteal artery leading to massive hemorrhaging (1), laceration of the lateral femoral cutaneous nerve resulting in loss of sensation over the lateral thigh $(1,2,4)$, injury of the superior cluneal (1) and ilioinguinal $(1,4)$ nerves leading to loss of sensation in the buttocks and groin, respectively, gluteal gait due to extensive stripping of the outer table muscles during surgery $(1,3)$, abdominal wall herniation (2-4) and perforation of the peritoneum (1) during the anterior approach to the inner table of the ilium.

Until now, there has only been one report of transient femoral nerve palsy as a complication of iliac crest bone graft harvesting (6) cited in any surgical literature. In that case report, transient femoral nerve palsy was described as a complication of postoperative administration of analgesia at the surgical site of an anterior iliac crest bone graft harvest. True femoral nerve palsy has been reported as a complication in deep pelvic and abdominal surgeries in which improper retraction and/or prolonged hyperextension of the hip may have caused a nerve compression syndrome (7-11). In addition, surgical patients on antithrombolytic therapy have experienced hemorrhage within the iliacus $(12-14)$ and iliopsoas $(15,16)$ 

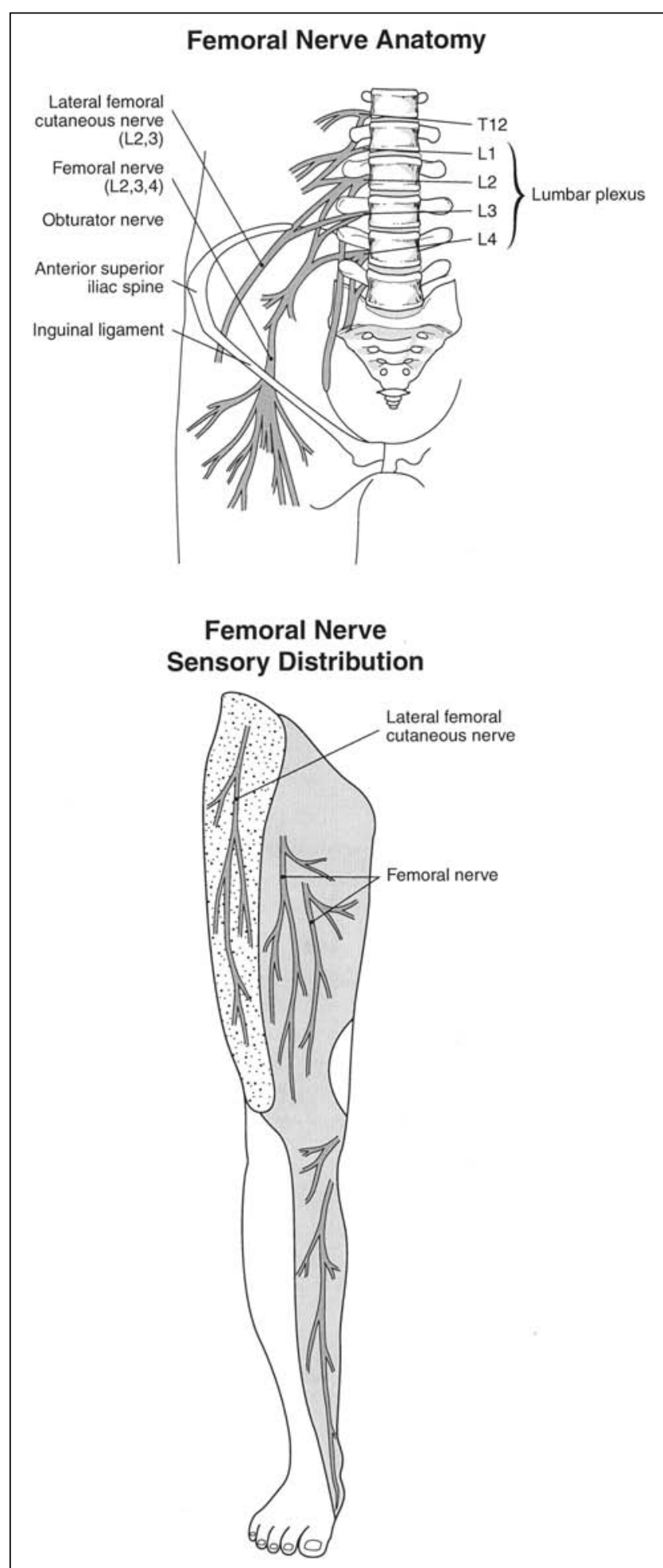

Figure 1) The femoral nerve is composed of branches from L2, L3 and L4. Sensory distribution to the lateral thigh is provided by the lateral femoral cutaneous nerve branch, while the femoral nerve supplies sensation to the anterior thigh and medial leg

muscles, leading to hematoma formation and femoral nerve compression. The femoral nerve (L2,3,4) provides sensation (Figure 1) to the anterior and medial thigh as well as the medial leg through the anterior femoral cutaneous, medial femoral cutaneous and long saphenous nerves, respectively. It

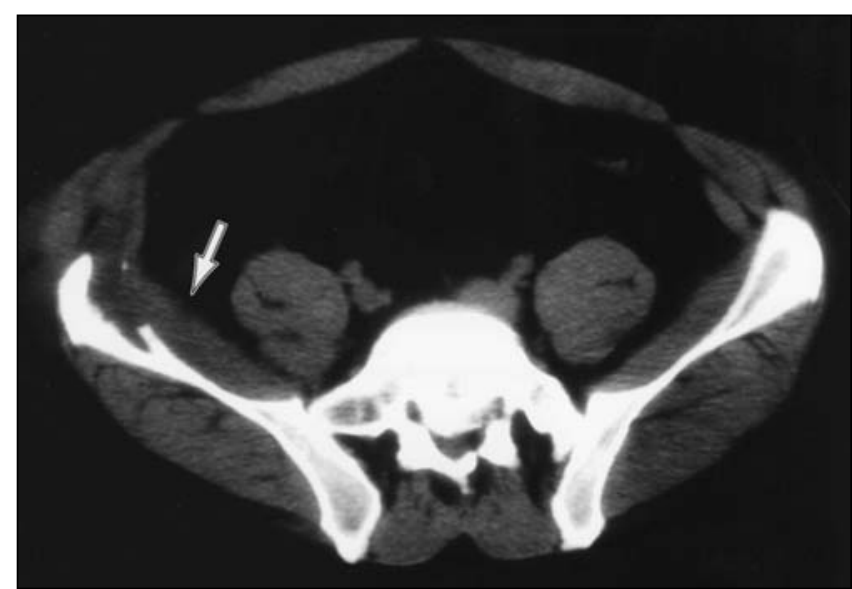

Figure 2) Computed tomography imaging of the lower abdomen performed on postoperative day 5 shows no evidence of hematoma formation (white arrow) at the level of the iliac crest bone harvest region or in the surrounding area

also supplies motor innervation to the quadriceps femoris, sartorius and pectineus muscles of the lower extremity. The classic motor and sensory deficits associated with femoral nerve palsy are reduced or absent patellar reflex, weakness with hip flexion and thigh extension, and anesthesia of the anterior thigh and medial aspect of the leg. Due to the serious nature of this complication, the operating surgeon should be aware of its potential presence, and the possibility of its occurrence should be included in the consenting process before surgery.

Two cases of femoral nerve palsy as a complication of anterior iliac crest bone graft harvesting are presented herein. The etiology of each is different, although the deficits are identical.

\section{Case 1}

\section{CASE PRESENTATIONS}

A healthy 40-year-old man underwent a LeFort I advancement and bone grafting procedure. A hip roll was initially placed for exposure of the anterior iliac crest, but was left in place throughout the entire surgery. A $4 \mathrm{~cm} \times 5 \mathrm{~cm}$ cortical-cancellous block graft and cancellous marrow was harvested in the usual manner using the medial approach technique. Hemostatic agents were applied and a drain was placed. The surgical wound was closed in multiple layers and the remainder of the surgery proceeded without incident.

On postoperative day 1, the patient complained of numbness and weakness in the right lower extremity. Patellar reflex was absent on examination. A computed tomography scan performed on postoperative day 5 (Figure 2) ruled out any hematoma. Electromyographic studies two months postoperatively revealed no conductive activity of the right quadriceps muscle. It was concluded that the patient had developed right femoral nerve palsy secondary to prolonged hip flexion or direct retractor injury during the initial bone harvest operation. This patient regained function of his right lower extremity, without residual sensory deficits, after several months of physical therapy and rehabilitation.

Case 2

A 47-year-old woman underwent right anterior iliac crest bone harvest for augmentation of her atrophic maxillary and mandibular alveolar ridges. The standard medial approach 
technique was used for the bone harvest. Postoperatively, the patient complained of right lower extremity weakness. Further investigation with a computed tomography scan on postoperative day 2 (Figure 3 ) revealed a retroperitoneal hematoma extending from the right iliac crest bone harvest area from the region of the iliacus to the right presacral space and lower retroperitoneum to the posterior perineal space, and between the layers of the right abdominal oblique muscles continuing externally to the right flank, the chest wall and subcutaneous fat overlying the gluteal muscles. This extensive hematoma was compressing the femoral nerve. The classic motor and sensory deficits of femoral nerve palsy were consistent with her complaints, and included flexion weakness of the thigh and leg and decreased sensation over the leg. Her condition completely resolved within six months with conservative therapy.

\section{DISCUSSION}

A review of the literature has shown that femoral neuropathy has only been reported as a complication in one case of iliac crest bone harvesting. Farrow et al (6) described a case report in which a bone harvesting procedure was performed without complication. In the immediate postoperative period, $0.25 \%$ bupivicaine was infused for $18 \mathrm{~h}$ via a $16 \mathrm{G}$ epidural cannula. Upon awakening, the patient complained of leg weakness and numbness consistent with a femoral nerve palsy, which continued until postoperative day 2 . The authors stated that femoral neuropathy is a widely recognized complication of regional anesthesia used in inguinal hernia repair, as well as urological, orthopedic and gynecological operations. They recommended that femoral nerve function should be tested before weightbearing in the immediate postoperative period, and should continue for up to $24 \mathrm{~h}$ following use of local regional anesthetic or local anesthesia in the ilioinguinal area.

Femoral neuropathy has also been reported as a complication of intrapelvic procedures, operations on hip joints, groin dissections and the use of retractors. Papastefanou et al (17) reported two cases of femoral nerve neuropathy as complications of anterior fusion. They concluded that this resulted from tight constriction of the nerve by a muscular portion of the psoas muscle when both the lumbar spine and the hip are immobilized in the position of maximum stretch of the muscle as is common in this operation. They recommended adequate flexion of the leg to eliminate this complication.

Our use of a hip roll in case 1, while not identical to an operation uing a flank approach, does have some shared similarities with regard to patient positioning. Both of the patients in the cited report complained of symptoms immediately postoperatively, and both experienced resolution in three to six months.

Hudson et al (18) reported on the treatment of 18 cases of iatrogenic femoral nerve injury. Thirteen of these patients had hip joint operations and all involved the use of a retractor inserted anterior to the hip joint and hooked over the brim of the pelvis. The incorrect placement of this retractor resulted in the nerve being compressed, causing fascicular damage of varying severity. Retractor injury could very well explain the complication in case 1 .

Nobel et al (19) researched the anatomical basis for femoral nerve palsy following iliacus hematoma. They stated that this entity has long been documented as a complication in hemophilia and occasionally seen as a consequence of sports injuries or motor vehicle accidents. Patients typically have swelling

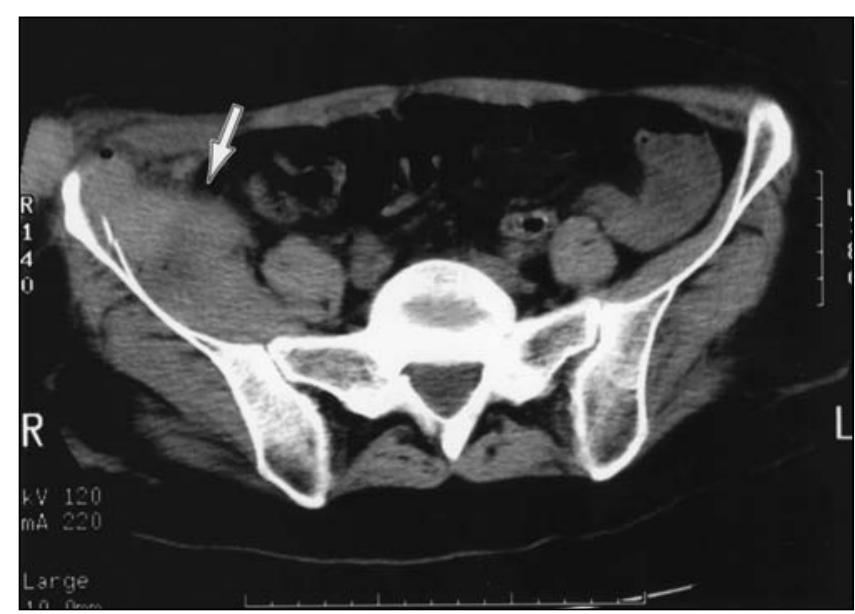

Figure 3) Computed tomography imaging of the lower abdomen performed on postoperative day 2 identifies a retroperitoneal hematoma (white arrow) at the level of the right iliac crest bone harvest region

due to hemorrhage in the iliac fossa. In their cadaver dissections, they identified up to four fascial layers parallel to the iliacus sheath. Up to three pouches were formed by variable states of fusion of these layers. These pouches were separated by loose connective tissue or fat. Latex was injected into the iliacus sheath and was seen to spread from the midlumbar region to the femoral triangle, resulting in compression and stretching and stretching of the femoral nerve in different parts of its course. They concluded that this was the anatomical basis for femoral nerve palsy during iliacus hematoma. This is a possible explanation for the complication in case 2 .

The authors recommend serial examinations, including electrophysiological studies, for evidence of return of motor, sensory and reflex function. The wound should be explored if there is no evidence of recovery by 14 weeks. At that time, the surgical team should be prepared to use electrophysiological testing and frozen section biopsies to assess the need for nerve grafting.

\section{CONCLUSION}

Although rare, this complication carries significant morbidity. Surgeons should discuss it with their patients preoperatively. In the medial approach to the iliac crest, retraction of the iliacus muscle should be judiciously performed. The hip roll, if it is needed at all, should certainly be removed once the bone harvest is completed. Finally, the cause of lower extremity sensory or motor deficits should not be automatically relegated to the lateral femoral cutaneous nerve or normal postoperative limitations. Femoral nerve dysfunction should always be considered in the differential diagnosis.

\section{REFERENCES}

1. Kurz LT, Garfin SR, Booth RE Jr. Harvesting autogenous iliac bone grafts. A review of complications and techniques. Spine 1989;14:1324-31.

2. Ahlmann E, Patzakis M, Roidis N, Shepherd L, Holtom P. Comparison of anterior and posterior iliac crest bone grafts in terms of harvest-site morbidity and functional outcomes. J Bone Joint Surg Am 2002;84-A:716-20.

3. Forrest C, Boyd B, Manktelow R, Zuker R, Bowen V. The free vascularised iliac crest tissue transfer: Donor site complications associated with eighty-two cases. Br J Plast Surg 1992;45:89-93. 
4. de la Torre JI, Tenenhaus M, Gallagher PM, Sachs SA. Harvesting iliac bone graft: Decreasing the morbidity. Cleft Palate Craniofac J 1999;36:388-90.

5. Silber, JS, Anderson G, Daffner SD, et al. Donor site morbidity after anterior iliac crest bone harvest for single-level anterior cervical discectomy and fusion. Spine 2003;28:134-9.

6. Farrow A, Morrison R, Pickersgill T, Currie R, Hammersley N. Transient femoral neuropathy after harvest of bone from the iliac crest. Br J Oral Maxillofac Surg 2004;42:572-4.

7. Kvist-Poulsen $\mathrm{H}$, Borel J. Iatrogenic femoral neuropathy subsequent to abdominal hysterectomy: Incidence and prevention. Obstet Gynecol 1982;60:516-20.

8. al Hakim M, Katirji MB. Femoral mononeuropathy induced by the lithotomy position: A report of 5 cases with a review of literature. Muscle Nerve 1993;16:891-5.

9. Burnett AL, Brendler CB. Femoral neuropathy following major pelvic surgery: Etiology and prevention. J Urol 1994;151:163-5.

10. Hall MC, Koch MO, Smith JA Jr. Femoral neuropathy complicating urologic abdominopelvic procedures. Urology 1995;45:146-9.

11. Kim DH, Kline DG. Surgical outcome for intra- and extrapelvic femoral nerve lesions. J Neurosurg 1995;83:783-90.
12. Stern MB, Spiegel P. Femoral neuropathy as a complication of heparin anticoagulation therapy. Clin Orthop Relat Res 1975;106:140-2.

13. Galzio R, Lucantoni D, Zenobii M, Cristuib-Grizzi L, Gadaleta A, Caffagni E. Femoral neuropathy caused by iliacus hematoma. Surg Neurol 1983;20:254-7.

14. Jamjoom ZA, al-Bakry A, al-Momen A, Malabary T, Tahan AR, Yacub B. Bilateral femoral nerve compression by iliacus hematomas complicating anticoagulant therapy. Surg Today 1993;23:535-40.

15. Mastroianni PP, Roberts MP. Femoral neuropathy and retroperitoneal hemorrhage. Neurosurgery 1983;13:44-7.

16. Niakan E, Carbone JE, Adams M, Schroeder FM. Anticoagulants, iliopsoas hematoma and femoral nerve compression. Am Fam Physician 1991:44:2100-2.

17. Papastefaneou SL, Stevens K, Mulholland RC. Femoral nerve palsy. An unusual complication of anterior lumbar interbody fusion. Spine 1994;19:2842-4.

18. Hudson AR, Hunter GA, Waddell JP. Iatrogenic femoral nerve injuries. Can J Surg 1979;22:62-6.

19. Nobel W, Marks SC Jr, Kubik S. The anatomical basis for femoral nerve palsy following iliacus hematoma. J Neurosurg 1980;52:533-40. 